\title{
Bicicletta e storia d'Italia (1870-1945). La modernizzazione su due ruote
}

\author{
di Eleonora Belloni
}

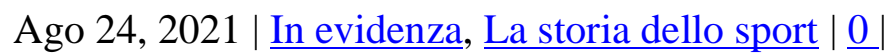

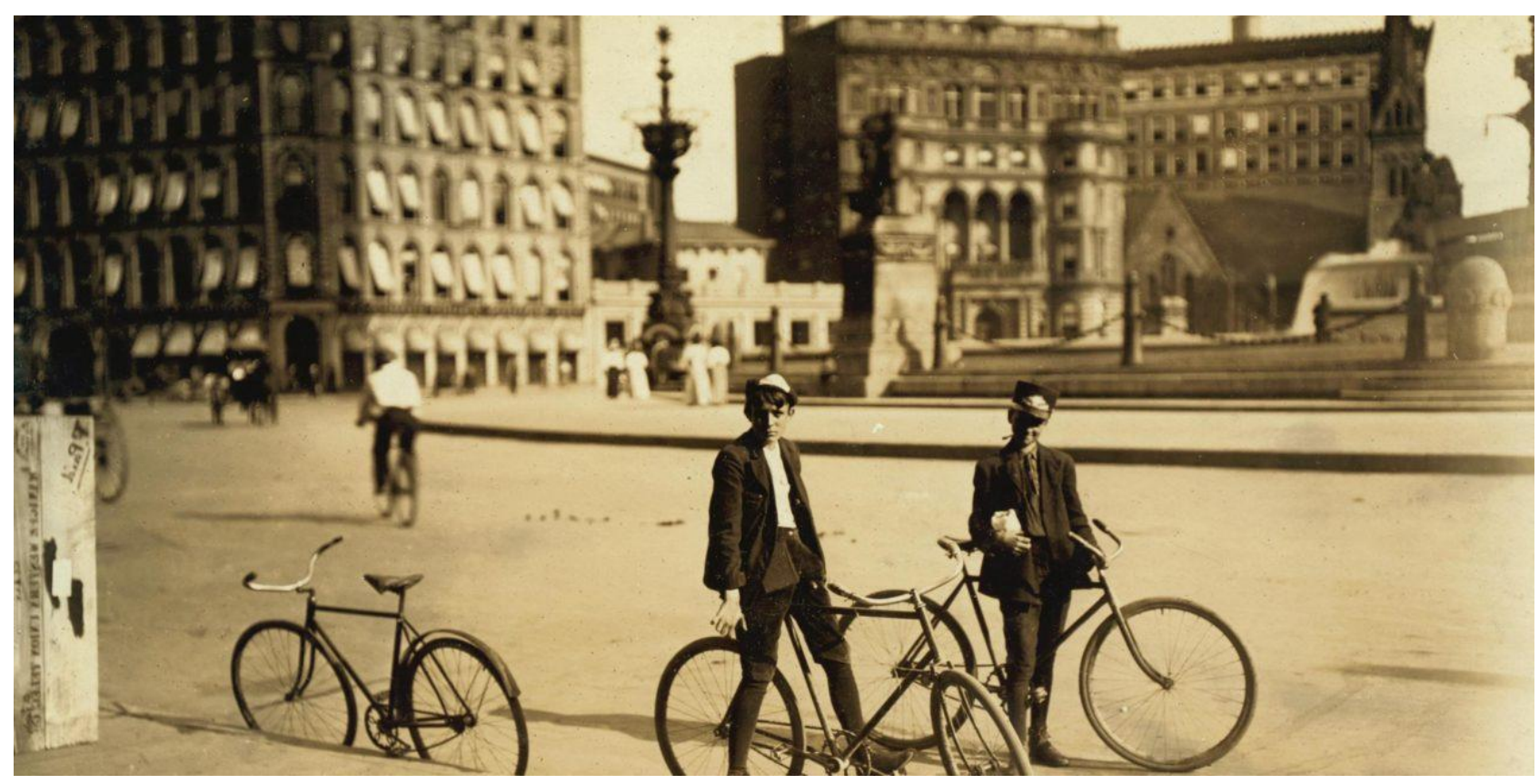

Foto tratta da $\underline{\text { Pixnio }}$

\begin{abstract}
Da quando la bicicletta fece la sua comparsa nelle strade italiane, negli anni Settanta dell'Ottocento, le sue vicende incrociarono quelle della modernizzazione della società. Partendo da questa premessa, il contributo vuole rileggere alcune delle tappe più importanti della vicenda storica nazionale attraverso la storia della due ruote. Una storia di successo, quella della bicicletta come mezzo di trasporto, come strumento di loisir, come fenomeno sportivo. Ma anche la storia di una lunga, combattuta e non sempre vittoriosa battaglia per la conquista dello spazio pubblico, in una contesa che non ha riguardato solamente il pedone, la bicicletta o l'automobile, ma ha chiamato in causa, di volta in volta, visioni diverse dello sviluppo, del progresso, della democratizzazione, del quadro culturale e valoriale di riferimento del percorso di costruzione dell'identità italiana.
\end{abstract}

\section{Bicicletta e modernizzazione}

La bicicletta fece la sua comparsa sulle strade italiane all'inizio degli anni Settanta dell'Ottocento, per poi diffondersi con relativa rapidità. Il fatto che la storia della sua prima diffusione coincida con il decollo industriale italiano ne fa un terreno di osservazione privilegiato per leggere il processo di modernizzazione del paese.

La storia della bicicletta, tanto per cominciare, è una pagina importante della storia dell'industrialismo italiano: l'industria del ciclo diviene ben presto una componente trainante di quell'industria meccanica che ha un ruolo centrale nel primo take off italiano, con un nucleo 
produttivo dove ai tanti laboratori artigianali già presenti all'inizio degli Ottanta dell'Ottocento si affiancano ben presto stabilimenti di grandi dimensioni, a partire da quella ditta Edoardo Bianchi che, sorta a Milano nel 1885, continua ancora oggi a portare la bicicletta italiana in giro per il mondo. Ma è anche storia del turismo e del tempo libero (due concetti a loro volta strettamente legati al processo di industrializzazione): con la bicicletta, e grazie all'associazionismo che si sviluppa attorno al nuovo mezzo, nasce un nuovo «genere» di viaggio e di modalità di scoperta del territorio, il cicloturismo. Ma la bicicletta intercetta anche processi politici (lo sviluppo dell'associazionismo) e sociali (l'emancipazione femminile) che a loro volta contribuiscono a comporre il quadro del processo di modernizzazione.

Non meno importante il fatto che, nel momento in cui fotografa il cammino dell'Italia verso la modernità, la storia della pratica ciclistica diviene anche storia del difficile rapporto del paese con quella modernità. Di questo rapporto controverso ci raccontano bene le tante resistenze, a cui in parte accenneremo, incontrate dalla bicicletta all'inizio del proprio cammino di diffusione.

\section{I pionieri del velocipedismo}

Gli anni Settanta dell'Ottocento segnarono anche in Italia il momento della prima istituzionalizzazione del ciclismo[1]. Questa fase pionieristica individua a sua volta due momenti ben distinti: uno, che possiamo definire «sportivo» (1870-1894), orientato molto precocemente alla competizione e alla ricerca del record. Anche in Italia l'esordio del ciclismo fu, infatti, sportivo ed elitario; la bicicletta attirò le brame dei giovani rampolli dell'alta società che iniziarono a sfidarsi, alla ricerca del brivido della velocità e dell'avventura, dapprima per gioco e poi in modo sempre più istituzionalizzato, in gare su pista e su strada. Il secondo momento, che possiamo definire «ricreativo» (1894-1900), in cui la funzione igienica e morale del velocipedismo - declinata in attività quali le passeggiate rigeneranti e le gite alla scoperta del territorio - finì per prevalere rispetto alla tensione agonistica fine a se stessa. A fare da spartiacque ideale tra i due momenti, la nascita del Touring club ciclistico italiano nel 1894. Ma andiamo per ordine...

Il 1870 segna idealmente l'inizio dell'avventura ciclistica nel nostro paese. In quell'anno infatti sorsero i primi due Veloce club della penisola: dapprima quello di Firenze (in quel momento capitale del Regno d'Italia), fondato nel gennaio (per la verità lo Statuto riporta come data il 7 dicembre 1869[2], ma l'atto costitutivo venne depositato il 15 gennaio), seguito dal Veloce club di Milano, nel marzo. A partire da quel momento i club velocipedistici si diffusero con relativa rapidità. Nel 1884 si contavano ormai 24 società, di cui 12 fondate solamente nel biennio 18831884[3]. Tre anni più tardi, nel 1887, le società risultavano più che raddoppiate (51)[4]; nel 1890 se ne contavano 62[5], nel 189264 e nel 1896 175[6].

La geografia di questo ciclismo pionieristico rispecchiava i confini dello sviluppo manifatturiero del Regno. La statistica del 1884 mostrava una mappatura che ricalcava quella del futuro triangolo industriale, con i club di Torino, Biella, Santhià, Milano, Como, Brescia, Mantova, Genova. I club più a sud risultavano quelli di Roma. Due eccezioni a questa mappatura geografica erano rappresentate dalla Toscana, che nel 1884 già contava cinque club (Firenze, Livorno, Pisa, Siena, Arezzo), e dal Veneto (Cerea, Padova, Treviso, Verona, Vicenza). Potevano quindi identificarsi, a metà anni Ottanta, tre macchie di diffusione geografica. Le statistiche successive mostrarono un ampliamento alla pianura padana (Bologna, Ferrara, Rimini) e al centro-sud (Ancona, Bari, Foligno, Napoli, Viterbo), ma la prima diffusione della pratica ciclista rimase un fenomeno che interessò una porzione molto limitata del territorio nazionale. È inoltre interessante notare che, fatta eccezione per la nascita del primo Veloce club nell'allora capitale del Regno, in seguito i luoghi di concentrazione 
del fenomeno ciclistico non coincisero mai con i centri politici del paese ma semmai con quelli economici.

La nascita di questi primi Veloce club rientra nella più generale diffusione del fenomeno associazionistico nell'Italia postunitaria, anche a seguito delle aperture legislative già avvenute con lo Statuto albertino del 1848, che all'art. 32 aveva riconosciuto la libertà di associarsi per finalità pacifiche e non politiche. Si trattava di un associazionismo regolato, dalle autorità statali e dai suoi stessi statuti, che proprio come tale veniva non solo tollerato ma anzi incoraggiato in quanto strumento di mediazione tra cittadini e Stato, capace di convogliare le spinte dal basso in forme controllate e controllabili di partecipazione, svolgendo anche una funzione di educazione civica e democratica.

I club velocipedistici ebbero inoltre un ruolo centrale nell'avviare la legittimazione sociale della pratica ciclistica. Oltre a divenire, con le loro sedi, luogo fisico di sociabilità e di incontro tra i praticanti, ebbero la funzione di regolamentare, attraverso i loro statuti, le attività. Gli statuti, infatti, oltre a contenere le classiche norme su scopi, diritti e doveri dei soci e funzionamento degli organi sociali, spesso erano accompagnati da regolamenti che dettavano veri e propri codici di comportamento per i soci, non solo riguardo alle corse (quasi sempre presente era la norma che vietava a coloro che partecipavano alle gare di riscuotere premi in denaro) ma anche sul contegno da tenersi in strada. Funzionavano, insomma, da piccoli codici stradali, colmando in tal senso le lacune normative. L'inserimento di questi regolamenti rispecchiava anche la preoccupazione che $\mathrm{i}$ conduttori di velocipedi non compromettessero con atteggiamenti imprudenti il modo in cui la loro presenza sulla strada era percepita dagli altri utenti, con il rischio di ostacolare il processo di legittimazione sociale del velocipede.

Iniziarono intanto le loro pubblicazioni alcuni periodici espressione più o meno diretta di questi soggetti rappresentativi degli interessi ciclistici: nel 1883 nasceva "La rivista Velocipedistica"; nel 1889 il foglio "Il Velocipede"; nel 1892 "La Illustrazione Velocipedistica Italiana", poi "La Illustrazione Ciclistica"; nel 1893 "Il Ciclo"; nel 1894 la "Gazzetta ciclistica" e "La bicicletta"; nel 1895 "La Tripletta" e la "Rivista mensile del Touring Club Ciclistico Italiano".

Il processo di istituzionalizzazione della fase pionieristica veniva infine completato dalla nascita dell'Unione velocipedistica italiana (Uvi), avvenuta a Pavia il 6 dicembre del 1885. Primo presidente fu Ernesto Nessi. La sede venne fissata dapprima a Como, per poi conoscere negli anni successivi diversi spostamenti (Torino, Genova, Alessandria, Milano) fino ad approdare a Roma nel 1929 (ma a quel punto l'Unione aveva intanto cambiato nome trasformandosi in Federazione).

Come da statuto (art. 1), scopi dell'Unione erano quelli di promuovere e regolamentare le corse ciclistiche, curare gli interessi dei velocipedisti nelle loro diverse attività, facilitare le relazioni e i buoni rapporti tra le diverse società ciclistiche[7].

\section{Il velocipedismo si organizza: la nascita del Touring club ciclistico italiano}

L'Unione tuttavia, a dispetto di quello che sembrava indicare il nome, non riuscì a legittimarsi come organo rappresentativo dell'intero panorama ciclistico nazionale, da subito attraversato da profonde divisioni interne. All'Unione velocipedistica italiana si opponeva infatti un gruppo di interesse che patrocinava il ciclismo nella sua forma di cicloturismo più che in quella strettamente sportiva, e che dopo il 1885 tentò più volte, ma con scarso successo, di creare una rappresentanza alternativa all’Uvi. Queste divisioni furono infine formalizzate con la nascita, nel 1894 a Milano, su iniziativa 
di Luigi Vittorio Bertarelli e Federico Johnson, del Touring club ciclistico italiano (Tcci)[8], che si aggiudicava la parte turistica della questione ciclistica[9], lasciando all'Uvi quella prettamente sportiva. Nel gennaio 1895 iniziava le sue pubblicazioni la "Rivista mensile del Touring Club Ciclistico Italiano"[10].

La realtà in cui il nuovo sodalizio si trovò ad agire contava ormai circa 175 tra società e club velocipedistici (di cui 99 rappresentati nell'Uvi)[11]. Il Tcci stesso conobbe una rapida espansione: i soci passarono dai 784 del 1894 ai 15.000 del 1898, per superare i 20.000 nel 1900[12].

Il nuovo sodalizio avviò un' attività su molteplici fronti: installazione di segnaletica stradale; firma di convenzioni con alberghi e meccanici per rendere più agevoli i viaggi dei soci; pubblicazione di itinerari, guide e carte stradali; studi per la manutenzione e il miglioramento delle strade; battaglie contro i regolamenti restrittivi e le imposizioni fiscali gravanti sulla bicicletta. Lo strumento più importante per l'attività di promozione ciclistica fu tuttavia rappresentato dalle escursioni che il Touring organizzò per mostrare, a soci e non, le potenzialità del mezzo a due ruote nel rivoluzionare la concezione stessa dello spostarsi sul territorio per motivi di svago o di utilità. L'escursione Milano-Roma del 1895 segnò in tal senso la prima, riuscita, dimostrazione: 70 partecipanti, divisi in due gruppi, partiti da Milano raggiunsero Roma rispettivamente in 4 e 7 giorni, dopo un viaggio in cui vennero messi alla prova tutti i nuovi servizi di assistenza garantiti dal sodalizio[13].

Ma la missione di cui il Tcci si fece carico fu qualcosa di più della pur importante attività logisticoorganizzativa. Il sodalizio si trovò infatti investito di un compito di legittimazione del nuovo mezzo in un panorama ancora dominato da un trasversale scetticismo che spesso degenerava in aperta ostilità, come del resto dimostrano i dati (che dal 1898 divenivano più attendibili e continuativi, a seguito dell'imposizione di una tassa nazionale sui velocipedi approvata l'anno precedente) che denunciano la ancora lenta diffusione della bicicletta nell'Italia di fine secolo.

Nel 1896 risultavano circa 30.000 biciclette circolanti sul territorio nazionale[14], salite a 109.019 nel 1899[15] e a 124.861 nel 1900[16]. In Francia, tanto per avere un termine di confronto,

all'inizio del 1895 ne risultavano circolanti più di 200.000. I dati del 1899 mostravano che dei quasi 110.000 velocipedi, 86.779 si trovavano nell'Italia settentrionale, 17.978 in quella centrale, $2.244 \mathrm{e}$ 2.018 rispettivamente in quella meridionale e in quella insulare[17].

Tab. 1 - Numero di biciclette circolanti in Italia e in Francia.

\begin{tabular}{lll}
\hline Anno & Biciclette circolanti in Italia & Biciclette circolanti in Francia \\
\hline 1895 & & 201.923 \\
1896 & 30.000 & 329.816 \\
1898 & $(185.000)^{*}$ & 483.414 \\
1899 & $109.019(200.000)$ & 838.556 \\
1900 & $124.861(215.000)$ & 975.878 \\
1901 & $142.918(221.000)$ & 1.106 .768 \\
\hline
\end{tabular}

Fonte: Belloni, 2019, p. 61. *Tra parentesi sono indicati dati non ufficiali, cioè non provenienti dalle statistiche sul pagamento della tassa sui velocipedi. Per questi dati cfr. G.G. Roseo, L'industria e il commercio dei velocipedi nel mondo, Libreria editrice milanese, Milano 1912, p. 223. 


\section{Tra modernità e conservatorismo}

Senza dubbio un freno importante alla diffusione del mezzo era rappresentato dal mancato sviluppo di una consolidata industria nazionale. È vero che nel 1885 era nata a Milano la ditta Edoardo Bianchi[18], destinata a divenire la più importante azienda nazionale del settore, preceduta da alcune piccole manifatture sorte sempre a Milano a partire dalla metà degli anni Sessanta dell'Ottocento. Ma ancora negli anni Novanta la produzione italiana appariva fortemente in ritardo rispetto a quella estera, e di conseguenza il mercato della due ruote continuava a essere marcatamente dipendente dalle importazioni, sia del prodotto finale che dei prodotti intermedi per l'assemblaggio. La dipendenza dalla produzione estera appare evidente se si guardano le pubblicità che occupavano le pagine delle riviste di settore, monopolizzate dalle ditte straniere, e ancor più se si leggono i cataloghi delle prime esperienze espositive dedicate al mezzo a due ruote.

Il ritardo industriale non era tuttavia l'unico fattore capace di spiegare la lenta diffusione della bicicletta in Italia. Non si deve infatti trascurare il ruolo che, in questa diffusione difficoltosa, ebbe il tipo di cultura che si andava costruendo attorno al nuovo mezzo. Come dimostrano le vicende legate al primo associazionismo ciclistico, l'identità creata dal comune interesse attorno alla bicicletta non era riuscita a costruirsi come identità unitaria, ma si era precocemente frammentata in diverse identità consolidate attorno a diversi modi di intendere la pratica ciclistica. Questo a sua volta rispecchiava divisioni sociali difficili da sanare. In tal senso, lo studio delle vicende legate al primo associazionismo ciclistico è molto utile perché aiuta a capire quale tessuto sociale fosse rappresentato nell'esperienza del velocipedismo pionieristico. I nomi dei promotori, dei presidenti e dei componenti gli organi direttivi dei club rimandano a una élite sociale che tuttavia spesso non era (o non soltanto) la vecchia élite aristocratica, quanto piuttosto la nuova élite alto-borghese legata al mondo degli affari, dei commerci e delle industrie emergenti. Una frattura ben rappresentata dalla dicotomia tra l'aristocrazia torinese e l'élite affaristica milanese e ben messa in scena dalla tensione che si era creata, come abbiamo visto, tra l'Unione velocipedistica e il nascente Touring. Una frattura, soprattutto, che rischiava di compromettere il progetto di una comunità nazionale costruita attorno alla pratica ciclistica.

L'iniziale diffusione del mezzo aveva inoltre fatto emergere i primi problemi di «convivenza». Il tema è ben evidenziato dalla questione dei regolamenti municipali che andavano a regolare la circolazione delle due ruote sulle strade, soprattutto urbane, dapprima proibendola quasi totalmente, poi regolandola, in modo spesso molto restrittivo. Quella che veniva configurandosi era una sorta di contesa tra i velocipedisti, da una parte, e le autorità municipali, dall'altra. Una contesa che molto precocemente si caratterizzò, o venne presentata, come una dicotomia tra i valori del progresso e della modernità, da una parte, e quelli della resistenza al progresso, magari sotto il camuffamento della tutela della sicurezza e del decoro, dall'altra. Le cronache del tempo sono ricche di episodi di ciclofobia, di volta in volta supportata da ragioni di prudenza e sicurezza o di dignità esteriore, che si traducevano poi in dispute generazionali, in battaglie di resistenza anti-secolarizzazione o in campagne igienico-sanitarie, spesso amplificate dalla stampa dell'epoca.

Ancora a fine secolo si moltiplicavano divieti e restrizioni, fatti rispettare da fin troppo zelanti agenti di polizia[19]. A Milano, ad esempio, nel 1869 il sindaco Giulio Belinzaghi aveva proibito «in via assoluta» la circolazione delle biciclette in alcune zone della città; nello stesso anno un provvedimento similare era stato adottato a Firenze. Persino l'avventurosa coppia anglo-americana che nel 1884 compì un tour in triciclo da Firenze a Roma dovette fare i conti con l'intransigenza delle guardie cittadine. In arrivo a Siena, le guardie alle porte della città intimarono ai coniugi di scendere dal triciclo e di continuare a piedi; a Roma i due coniugi dovettero dapprima scendere dal 
triciclo, poi comparire davanti al giudice che li condannò a pagare una multa di dieci franchi per guida pericolosa[20]. I divieti che si potevano leggere nei provvedimento municipali erano tra i più svariati, a volte ai limiti del surreale: dal divieto per i velocipedisti di percorrere il viale della stazione mezz'ora prima e mezz'ora dopo l'arrivo dei treni, a quello di circolare nei giardini pubblici e nelle vie del centro (perché troppo affollate), ma anche in quelle periferiche (perché troppo isolate), fino all'obbligo di portare al braccio una fascia nera di riconoscimento[21].

\section{Questioni di «decoro»}

La bicicletta non era ritenuta solamente pericolosa, per gli stessi velocipedisti e per gli altri utenti della strada. In molti casi fu anche considerata sconveniente. Lo era, soprattutto, per tutti coloro il cui ruolo pubblico, o semplicemente sociale, sconsigliava atteggiamenti, pose, abiti suscettibili di sollevare scandalo. Ecco allora che nella rete del proibizionismo perbenista di fine secolo caddero ufficiali dell'esercito, rappresentanti dell'ordine pubblico e, soprattutto, donne ed esponenti del clero.

A smorzare gli entusiasmi di quei sacerdoti - soprattutto parroci di campagna - che avevano precocemente colto le potenzialità che il nuovo mezzo poteva avere per spostarsi più rapidamente sul territorio arrivarono, sotto il papato di Leone XIII, i primi pronunciamenti ufficiali da parte di vescovi, cardinali e organismi vaticani contro la diffusione del nuovo mezzo, anche e soprattutto tra i sacerdoti[22].

Ancor più agguerrite furono le resistenze nei confronti dell'uso della bicicletta da parte delle donne[23]. Se, infatti, gli scetticismi che da subito avevano circondato le prime temerarie donne velocipediste erano gli stessi che più in generale riguardavano la donna che voleva approcciarsi allo sport - con un'unica parziale eccezione per la ginnastica - nel caso della bicicletta le resistenze erano rafforzate dall'idea di libertà, anche spaziale, associata al mezzo a due ruote. Le donne in sella alla loro bicicletta potevano sottrarsi più facilmente al controllo esercitato da padri, fratelli, mariti; così come più facilmente potevano trovare occasioni di promiscuità con individui di sesso maschile. Eppure, proprio grazie a quell'idea di libertà che portava con sé, la bicicletta esercitò da subito un grande fascino sul genere femminile.

Dei primi anni Novanta del XIX secolo sono le notizie, provenienti soprattutto da Milano, di donne iscritte a gare ciclistiche. Nel 1893, in particolare, si ricorda sia la sfida tra la cantante e attrice Lina Cavalieri e la fioraia Adelina Vigo, sia la partecipazione di Maria Forzani a una gara maschile di 60 chilometri. Altro nome quello di Alessandrina Maffi, che vinse a più riprese gare contro ciclisti uomini, fino ad aggiudicarsi il premio Audax di velocità nel 1898[24]. Mentre dall'Austria, dal Belgio e dalla Germania arrivavano le notizie dei primi club velocipedistici interamente femminili, in Italia quasi immediata arrivò la risposta dell'Unione velocipedistica italiana, che nel 1894 sanciva il divieto per le donne di partecipare a gare su pista e su strada[25].

Se la donna in bicicletta stentava dunque a trovare spazio sulla strada e nelle competizioni sportive, al contrario ben presto invase le pagine delle riviste e i manifesti pubblicitari. Quello del binomio donna-bicicletta divenne un vero e proprio stereotipo della cartellonistica pubblicitaria di inizio Novecento[26]. A dispetto delle forti resistenze, dunque, l'immagine sublimata della donna, elegante e alla moda, in sella alla due ruote venne utilizzata come strumento per attirare le attenzioni sul nuovo mezzo a scopo pubblicitario. Un messaggio che era rivolto sia agli uomini, che si presumevano attirati dalle immagini eleganti e a volte ammiccanti delle belle cicliste, sia alle donne stesse, che precocemente l'industria pubblicitaria intuì potessero rappresentare uno dei bacini 
di mercato su cui fare maggiormente leva. Le stesse ditte produttrici, del resto, si erano affrettate ad aggiungere ai loro cataloghi modelli «da donna», con piccoli accorgimenti sulle selle e sui telai in grado di scongiurare i reali o presunti problemi generati alle donne dalla pratica ciclistica.

\section{La democratizzazione della bicicletta}

All'inizio del nuovo secolo la bicicletta stava divenendo un mezzo di trasporto quotidiano per gli spostamenti, verso il luogo di lavoro o per raggiungere le città vicine dalle campagne[27]. Una serie di professioni potevano trovare nella bicicletta vantaggi in termini di risparmio di fatica e di tempo: medici, guardie cittadine e agenti di polizia, pompieri, fattorini postali e telegrafici[28]. Uno dei fattori che favorì questa maggiore democratizzazione della due ruote fu sicuramente la riduzione dei prezzi, accompagnata da un sempre più fiorente mercato di mezzi di seconda mano. Se alla fine dell'Ottocento il prezzo di una bicicletta si aggirava attorno alle 600-700 lire (a fronte di un salario giornaliero di circa 1,69 lire), nel 1905 il prezzo era già sceso attorno alle 200 lire per una bicicletta nuova di buona qualità, ma di fatto era possibile acquistare mezzi di minore qualità o di seconda mano anche a prezzi tra le 40 e le 100 lire[29]. Questo fu a sua volta reso possibile da uno sviluppo dell'industria nazionale della bicicletta e da una maggiore standardizzazione della produzione.

Tab. 2 - Numero di biciclette prodotte in Italia.

\begin{tabular}{ll}
\hline Anno & Biciclette prodotte in Italia \\
\hline 1907 & 98.062 \\
1908 & 131.100 \\
1909 & 169.900 \\
1910 & 221.612 \\
1937 & 400.000 \\
\hline
\end{tabular}

Fonte: Belloni, 2019, p. 78.

Anche i primi utilizzi delle biciclette a scopi militari[30] ebbero sicuramente un ruolo non secondario nel legittimare l'idea e la pratica utilitaria del mezzo a due ruote, così come alla democratizzazione della bicicletta contribuirono le prime aperture da parte del movimento socialista, inizialmente scettico verso una pratica identificata come propria della sociabilità borghese. Promotore della rivalutazione della due ruote da parte del movimento socialista fu Ivanoe Bonomi, che non esitò a definire la bicicletta «un nuovo simbolo di democrazia»[31]. Nel 1912 nascevano a Imola $i$ «ciclisti rossi», non a caso nel cuore di quella pianura padana che già ad inizio Novecento faceva registrare le percentuali più elevate di diffusione della bicicletta in rapporto alla popolazione. Al gruppo di Imola ne seguirono poi molti altri nei mesi successivi, soprattutto in pianura Padana ma anche nel resto dell'Italia centrale e settentrionale, fino alla costituzione della Federazione nazionale dei ciclisti rossi avvenuta sempre a Imola il 10 agosto 1913. Nell'apertura al movimento ciclistico da parte del socialismo gli scopi pratici prevalevano su quelli ideali. I ciclisti rossi erano chiamati a mobilitarsi con funzione di collegamento in occasione di scioperi e agitazioni, organizzando dei veri e propri servizi di staffetta che anticipavano quanto sarebbe accaduto con le staffette partigiane. Ma tra le battaglie portate avanti dal movimento vi era anche quella per la diffusione democratica di biciclette economiche tra le masse lavoratrici, in un 
momento in cui il mezzo a due ruote, come abbiamo visto, iniziava a diffondersi ma con fatica, rimanendo facilmente accessibile soprattutto ai maschi della media e alta borghesia dell'Italia settentrionale. A Milano alcune case produttrici, sensibili ai richiami del ciclismo rosso, iniziarono a pubblicizzare biciclette «Avanti!» montanti pneumatici «Carl Marx», le uniche che gli aderenti al movimento per statuto erano autorizzati a condurre, possibilmente indossando una maglia rossa come segno distintivo e identitario[32].

Fu, quella della bicicletta, una popolarizzazione che i numeri aiutano a cogliere nel suo aspetto quantitativo:

Tab. 3 - Numero di biciclette circolanti in Italia.

\begin{tabular}{ll}
\hline Anno & Biciclette circolanti in Italia \\
\hline 1901 & 142.918 \\
1907 & 368.181 \\
1910 & 930.651 \\
1914 & 1.250 .701 \\
1920 & 1.603 .569 \\
\hline
\end{tabular}

Fonte: Belloni, 2019, p. 88.

\section{Arriva il Giro}

Tra le ragioni di popolarizzazione della due ruote nell'Italia di inizio Novecento non si può trascurare il ruolo giocato dal ciclismo sportivo e, in particolare, dalla nascita del Giro d'Italia[33].

La prima gara su strada in Italia viene comunemente ritenuta la Firenze-Pistoia del 1870 , corsa all'indomani della fondazione del Veloce club fiorentino[34]. Nell'età pionieristica, in realtà, furono le gare su pista a generare i maggiori entusiasmi: gare di velocità, quindi, più che di resistenza. Solo a partire dalla fine del XIX secolo le gare su strada iniziarono a moltiplicarsi. Ai primi anni del XX secolo risalgono alcune competizioni divenute poi «classiche»: nel novembre del 1905 Armando Cougnet e Tullo Morgagni idearono, assieme a "La Gazzetta dello Sport", il Giro di Lombardia (230 chilometri nella prima edizione), che doveva funzionare da grande evento per lanciare l'Esposizione di Milano dell'anno successivo e che sarebbe poi divenuta la classica d'autunno, a chiusura della stagione ciclistica; nell'aprile del 1907 partiva invece la prima edizione della Milano-Sanremo, altra gara destinata a divenire una classica di primavera. Nel frattempo era stato corso anche il primo Giro del Piemonte, nel 1906. Furono queste prove su strada a consacrare i primi eroi del pedale: da Romolo Buni a Federico Momo e Gian Fernando Tomaselli fino al «Diavolo Rosso» Giovanni Gerbi.

La maggior parte di queste competizioni nacque all'interno del circuito de "La Gazzetta dello Sport", già affermatasi in quegli anni come il più popolare giornale sportivo italiano, dopo essere stata fondata ormai oltre un decennio prima, nel 1896, a seguito della fusione di due preesistenti fogli ciclistici, "Il Ciclista" e "La Tripletta". 
Non possiamo, infine, non ricordare che nel luglio del 1903 era stata corsa, su iniziativa di Henri Desgrange e del quotidiano sportivo "L'Auto", la prima edizione del Tour de France, quasi 2.500 chilometri di percorso con partenza e arrivo a Parigi, vinta da Maurice Garin.

La spettacolarità e il successo del Tour ebbero un ruolo centrale nel mettere in moto anche in Italia il processo che avrebbe portato, sei anni più tardi, al primo Giro d'Italia, partito da Milano il 12 maggio del 1909. Gli intenti dei promotori della corsa erano chiari: favorire la vendita di un oggetto - la bicicletta - simbolo di uno dei settori trainanti della nascente economia industriale; incrementare la tiratura de "La Gazzetta dello Sport" (trasformatasi in quell'anno da bisettimanale a trisettimanale, poi dal 1913 quotidiano), promotrice dell'evento; accrescere la diffusione e la popolarità del ciclismo.

Come nel caso del Tour, anche per il Giro d'Italia fu subito evidente il legame che univa a doppio filo evento sportivo, circuito mediatico e apparato produttivo nazionale. Non a caso a sostenere l'iniziativa dei tre ideatori - Eugenio Camillo Costamagna (direttore della Gazzetta), Armando Cougnet e Tullo Morgagni - vi fu fin da subito Angelo Gatti, industriale dell'Atala intenzionato a imporre i propri velocipedi sul mercato bruciando la concorrenza di Edoardo Bianchi, sostenitore di un'analoga iniziativa promossa dal "Corriere della Sera" (e sostenuta dal Touring) e che solo per poco venne anticipata da quella, vincente, della rosea. Il ciclismo era stato del resto anche il primo sport a riconoscere precocemente, nel 1890, la liceità del professionismo tra i suoi praticanti. La prima edizione avrebbe assegnato un totale di 70.000 lire di premi (offerti da "La Gazzetta dello Sport", "Corriere della Sera", Pirelli, Uvi e industrie ciclistiche), di cui 18.900 spartiti tra i corridori classificatisi nella graduatoria finale (5.325 andarono al vincitore Luigi Ganna).

In effetti, il primo Giro non deluse le attese dei suoi organizzatori: 127 partecipanti (gli iscritti inizialmente erano 166, gran parte dei quali provenienti dal nord Italia, soprattutto area lombarda, seguiti da piemontesi, romagnoli, toscani e veneti); folle assiepate lungo tutto il percorso; "La Gazzetta" che vide accrescere le sue tirature oltre le 100.000 copie. Lo stesso aumento delle biciclette circolanti nel paese, che quasi raddoppiarono tra il 1909 e il 1910, risentì senza dubbio dell' «effetto Giro», oltre che della riduzione della tassa di circolazione, passata in quell'anno da 10 a 6 lire.

\section{La concorrenza dell'automobile}

La bicicletta non aveva ancora concluso il suo percorso di legittimazione quando iniziò a essere minacciata dal rapido avanzare di una temibile concorrente: l'automobile. Il Touring club ciclistico italiano fu rapido a cogliere il cambiamento in atto, e già nel 1900 decise di cambiare il suo nome in Touring club italiano, a sottolineare che la sua attività di promozione sarebbe stata rivolta al turismo e alla scoperta del territorio esercitati con qualsiasi mezzo, compresi quelli motorizzati[35]. Così facendo, tuttavia, il sodalizio privava di fatto la pratica ciclistica di una rappresentanza esclusiva dei propri interessi.

Il Novecento si annunciava dunque come «il secolo dell'automobilismo». Eppure, ancora per molti anni, l'automobile sarebbe apparsa tutt'altro che concorrenziale rispetto non solo alla bicicletta, ma anche ai trasporti pubblici come il treno. Sia per i costi, sia perché la situazione delle strade rendeva i tempi di percorrenza molto lunghi. Ad esempio, il tratto Napoli-Roma veniva percorso in circa sei ore contro le due e mezza del treno; il tratto Milano-Genova poteva richiedere anche un'intera giornata contro le tre ore del treno[36]. 
L'automobile divenne, dunque, simbolo di modernità e di libertà contro ogni evidenza di praticità e di attuabilità. E attorno a essa iniziò a costruirsi quell'immaginario che ne avrebbe fatto il simbolo della rivoluzione della mobilità del XX secolo. Parallelamente, i ciclisti, assieme ai pedoni, iniziavano a essere associati all'immagine di qualcosa di «popolare» e «modesto», mentre erano gli automobilisti ad appropriarsi dell'aura di modernità e di velocità che fino a pochi anni prima era stata appannaggio della due ruote.

In questo quadro, la Grande guerra ebbe senza dubbio un ulteriore effetto propulsivo sull'industria automobilistica, che si era andata affermando come una delle voci principali nelle esportazioni italiane; ma lo ebbe anche sulla stessa industria ciclistica, che aveva visto la sua attività accresciuta per soddisfare non solo la domanda civile ma anche quella militare. Tra l'altro, il minore controllo maschile, da una parte, e l'esigenza di spostarsi con maggiore facilità per adempiere ai nuovi compiti familiari e lavorativi, dall'altra, avevano favorito un maggiore accesso anche da parte delle donne all'utilizzo del mezzo a due ruote. Per tutti questi motivi, gli anni dell' immediato dopoguerra videro accrescersi il numero di biciclette in circolazione, anche grazie all'immissione sul mercato di quelle che erano appartenute all'esercito e che si erano salvate dalle distruzioni o dall'usura degli anni di guerra.

Sempre più numerose, dunque, le biciclette, ma allo stesso tempo sempre più marginalizzate in una città pianificata a misura di automobile. Iniziava infatti a delinearsi con chiarezza l'idea di una gerarchia nel diritto di accesso alla circolazione stradale, dove il pedone veniva relegato ad essere «il bastone tra le ruote dell'ordine circolatorio»[37]. Ma anche la bicicletta appariva sempre più marginalizzata, e i suoi utenti costretti a fare i conti con un numero crescente di divieti, limitazioni, casi di furti non adeguatamente puniti, strutture pubbliche e private che si rifiutavano di accogliere le biciclette nei loro androni. La presenza di veicoli diversi, e capaci di garantire diverse velocità di movimento, sulle strade era additata come causa principale dei disagi della circolazione. È ovvio come l'idea che stava alla base di questo progetto di circolazione «ordinata» fosse quella di una strada dominata dall'automobile. A guidare questo movimento modernizzatore veniva posta una nuova classe di ingegneri e tecnici, con un occhio privilegiato a quanto si faceva oltreoceano. Nel 1918 nasceva l'Istituto sperimentale per materiali stradali, finanziato dall'ingegnere milanese Piero Puricelli e posto sotto la direzione di Italo Vandone, proprio con lo scopo dichiarato di affrontare il problema del miglioramento stradale, che diveniva dunque centrale nell'agenda del Touring del dopoguerra.

\section{La bicicletta di massa}

Quando il fascismo arrivò al potere le biciclette circolanti in Italia erano ormai quasi due milioni (1.849.000), contro poco più di 40.000 automobili[38]. Nel 1935 - un compleanno importante per la bicicletta, che festeggiava i cinquant'anni dalla messa in circolazione del modello «moderno», la safety bicycle - il numero era più che raddoppiato (3.992.000). Se ne sarebbero contate addirittura poco meno di cinque milioni (4.935.000) nel 1938, nell'ultima statistica attendibile, prima che la definitiva abolizione della tassa sui velocipedi, avvenuta in quell'anno (con entrata in vigore dal gennaio 1939), facesse venir meno questo strumento indiretto di rilevazione.

Tab. 4 -Numero di biciclette, motocicli e automobili circolanti in Italia. 


\begin{tabular}{llll}
\hline Anno & Biciclette & Motocicli & Autovetture \\
\hline 1922 & 1.849 .272 & 35.751 & 41.035 \\
1925 & 2.549 .718 & 62.801 & 84.826 \\
1930 & 4.480 .000 & 71.652 & 183.292 \\
1938 & 4.935 .019 & 142.094 & 289.174 \\
1941 & 5.000 .000 & 17.175 & 97.616 \\
\hline
\end{tabular}

Fonte: Belloni, 2019, p. 137.

Tra le due guerre, insomma, milioni di italiani viaggiavano ormai su due ruote per le lor esigenze quotidiane di spostamento. La democratizzazione del mezzo poteva ormai dirsi compiuta, le resistenze alla sua diffusione in gran parte superate, l'emancipazione produttiva dall'estero ormai quasi totalmente realizzata.

Se i numeri ci dicono che la bicicletta dominava le strade degli anni Venti e Trenta, la propaganda fascista pretendeva però di presentare agli italiani e al mondo una realtà totalmente diversa: il mezzo del futuro era l'automobile, e il fascismo modernizzatore l'avrebbe resa accessibile a tutti. La bicicletta veniva dunque sacrificata, nell'opera di propaganda e di costruzione del modello di mobilità, all'automobile, che passava da essere bene di lusso e strumento delle vittorie sportive sui circuiti internazionali a mezzo di trasporto sempre più «per tutti». Il primo segnale importante della netta sterzata a favore dell'automobile fu rappresentato dai progetti per l'autostrada Milano-laghi, inaugurata il 21 settembre del 1924. Il progetto, opera dell'ingegnere Puricelli, fu portato avanti dall'omonima Fondazione e ricevette il pieno appoggio del Touring. L'aspetto più innovativo del progetto stava proprio nel prevedere una rete stradale «esclusivamente automobilistica», come del resto denunciava il nome stesso[39]. La trasformazione nel 1927 dell'Automobile Club d'Italia in ente parastatale attraverso la costituzione del RACI (Reale Automobile Club Italia) e la creazione nello stesso anno della Milizia della strada, un corpo di camicie nere adibite al controllo del traffico, formalizzavano di fatto la scelta del regime di indirizzare e controllare la politica automobilistica della Nazione, completata dal controllo statale sulle strade ordinarie attraverso la costituzione dell'Azienda Autonoma Statale della Strada (AASS) nel 1928.

A partire dalla metà degli anni Trenta, tuttavia, il regime si trovò costretto a recuperare la bicicletta in funzione autarchica. Quella a cui si assistette fu una vera e propria riabilitazione della due ruote, che tornava a essere valorizzata come mezzo di trasporto in grado di garantire un risparmio in termini di consumo di carburante e di manutenzione stradale, ma anche come possibile alleata dei progetti di ruralizzazione del paese. Mezzo autarchico anche nella produzione, con alcuni esperimenti, per la verità di scarso successo, di mezzi in cui il telaio metallico era sostituito da telai in composti plastici o interamente costruiti in legno (la Littorina autarchica)[40]. L'abolizione della tassa sui velocipedi non fu, dunque, che il punto culminante di questo processo di recupero autarchico della bicicletta.

Un recupero che avveniva, tuttavia, non nel quadro di una ragionata e consapevole politica della mobilità favorevole alle due ruote, ma all'interno della cornice delle contraddizioni della politica economica fascista, che da una parte si presentava come portatrice di modernizzazione e di progresso, dall'altra si trovò a fare i conti con una crisi economica e con un isolamento internazionale che sembravano necessariamente convergere verso una politica autarchica dove ai temi della modernità e della velocità si opponevano quelli del risparmio, del sacrificio, della valorizzazione delle risorse interne. In tal modo la bicicletta finiva per essere inevitabilmente 
associata a un'idea di austerità, di povertà, di sacrificio che ne avrebbe irrimediabilmente compromesso l'immagine sociale e, in ultima analisi, il rapporto culturale, e di conseguenza materiale, con gli italiani.

\section{Note:}

[1] Per tutto quello che segue, dove non diversamente indicato, mi permetto di rimandare a E. Belloni, Quando si andava in velocipede. Storia della mobilità ciclistica in Italia (1870-1955), FrancoAngeli, Milano 2019, pp. 39-74.

[2] Veloce-club di Firenze. Statuto, Tipografia Eredi Botta, Firenze 1870. Sulla nascita del ciclismo fiorentino cfr. E. Belloni, La nascita della Toscana "ciclistica". Dagli esordi alla Grande guerra, in "Rassegna storica toscana", LXVI, 2, 2020, pp. 235-257.

[3] Statistica delle società italiane nell'agosto 1884, in "La rivista velocipedistica", n. 15-16, 1-15 settembre 1884, pp. 82-84.

[4] Società Velocipedistiche Italiane, in "La rivista velocipedistica", n. 93-94, 15 dicembre 1887-1 ${ }^{\circ}$ gennaio 1888 , p. 896.

[5] Società Velocipedistiche Italiane, in "La rivista velocipedistica", n. 153-154, 1-15 gennaio 1891, p. 2340.

[6] Elenco delle Società iscritte nella UVI al 15 febbraio 1892. Società velocipedistiche italiane non iscritte nella UVI, in "Unione Velocipedistica Italiana. Bollettino Ufficiale per gli atti e comunicazione della Unione”, n. 44, gennaio-febbraio-marzo 1892, pp. 234-237; V. Monaco, Il velocipedismo, Ditta Nicola Zanichelli, Bologna 1896.

[7] Statuto, in "Unione Velocipedistica Italiana. Bollettino Ufficiale per gli atti e comunicazione della Unione", n. 11, marzo 1888, pp. 8-12.

[8] Sul Touring si vedano: G. Vota (a cura di), I sessant'anni del Touring Club Italiano 1894-1954, Touring Club Italiano, Milano 1954; R.J. Bosworth, The Touring Club Italiano and the nationalization of the Italian bourgeoisie, in "European history quarterly", 27(3), 1997, pp. 371410; D. Bardelli, L'Italia viaggia. Il Touring Club, la nazione, la modernità, 1894-1927, Bulzoni, Roma 2004; S. Pivato, Il Touring Club Italiano, Il Mulino, Bologna 2006.

[9] Sul rapporto tra pratica ciclistica e turismo cfr. S. Maggi, Ciclismo e turismo, in G. Silei (a cura di), Il Giro d'Italia e la società italiana, Lacaita, Manduria-Bari-Roma 2010, pp. 79-99.

[10] La rivista, che avrebbe poi più volte cambiato nome nel corso degli anni, è adesso in gran parte consultabile online grazie alla digitalizzazione realizzata dal Touring, e che ha interessato, oltre ad alcune delle pubblicazioni periodiche, anche una parte del patrimonio fotografico. Grazie a questa operazione la documentazione messa a disposizione rappresenta una fonte preziosa non solo per lo studio della storia del sodalizio, ma anche per gli studi relativi alla storia della mobilità, del turismo, del territorio. La documentazione digitalizzata è disponibile all'indirizzo https://www.digitouring.it/. 
[11] Monaco, 1896, p. 147.

[12] Bardelli, 2004, p. 130.

[13] Vota, 1954, pp. 47-48.

[14] A sua eccellenza il Ministro delle Finanze, in "Rivista mensile del Touring Club Ciclistico Italiano", n. 2, febbraio 1896, pp. 18-22.

[15] L.V. Bertarelli, Statistica Istruttiva, in "Rivista mensile del Touring Club Ciclistico Italiano", n. 1, gennaio 1900, pp. 8-9.

[16] Quanti sono i ciclisti in Italia, in "Rivista mensile del TCI”, n. 3, marzo 1903, p. 98.

[17] Bertarelli, 1900.

[18] Sulla storia della Bianchi si possono vedere: L'industria della bicicletta a Milano dalla fine dell'Ottocento al 1914, in "Storia in Lombardia", a. IV, n. 2, 1985, pp. 81-99; C. Mari, Putting the Italians on bicycles: marketing at Bianchi, 1885-1955, in "Journal of Historical Research in Marketing”, vol. 7, n. 1, 2015, pp. 133-158; D. Marchesini, Bianchi. Una storia italiana, Bolis edizioni, Azzano San Paolo 2018, C. Mari, A Business History of the Bicycle Industry. Shaping Marketing Practices, Palgrave Pivot, 2021.

[19] Cfr. Sunto dei regolamenti municipali sull'uso dei velocipedi in alcune città d'Italia, in "Unione Velocipedistica Italiana. Bollettino Ufficiale per gli atti e comunicazione della Unione", a. V, n. 44, gennaio-febbraio-marzo 1892, pp. 237-239.

[20] Joseph Pennell ed Elisabeth Robins raccontarono il loro viaggio in un libro uscito nel 1887, An Italian Pilgrimage, poi più volte tradotto in italiano. Cfr. J. Pennell e E. Pennell, L'Italia in velocipede, Sellerio editore, Palermo 2002.

[21] L. Gazzaniga, Cicloturismo d'oggi e di ieri, in "Le vie d'Italia", maggio 1948, pp. 439-444.

[22] Sulle vicende del rapporto contrastato tra clero e bicicletta cfr. S. Pivato, Storia sociale della bicicletta, Il Mulino, Bologna 2019, pp. 47-64.

[23] Ivi, pp. 65-84. Sul complesso rapporto tra donne e bicicletta si può vedere anche A. Stelitano, Donne in bicicletta. Una finestra sulla storia del ciclismo femminile in Italia, Ediciclo editore, Portogruaro 2020, pp. 23-81.

[24] Cfr. G. Gori, Muliebris birota velocissima. Il ciclismo femminile in Italia tra XIX e XX secolo, in A. Cagnolati (a cura di), Donne e bicicletta: una relazione pericolosa?, Aracne, Roma 2011, pp. 63-88.

[25] “Gazzetta ciclistica”, a. I, n. 12, 29 dicembre 1894, p. 149.

[26] A. Cagnolati, Donne in bicicletta. La pubblicità nella Belle Époque, in Cagnolati, 2011, pp. 89-107; Due ruote, cento manifesti: la bicicletta nei cartelloni pubblicitari della Raccolta Salce. Treviso, Museo Civico “L. Bailo”, 20 giugno-6 ottobre 1985, Treviso 1985.

[27] Per quanto segue cfr. Belloni, 2019, pp. 75-110. 
[28] La proposta di utilizzo della bicicletta come mezzo di cui fornire i postini, soprattutto nelle zone rurali, era stata avanzata per la prima volta in Parlamento nel 1895 dal ministro delle Poste e Telegrafi Maggiorino Ferraris. Cfr. Atti Parlamentari, Camera dei Deputati. Discussioni, Legislatura XIX, Tornata del 29 giugno 1895, pp. 429-430.

[29] Archivio storico del Touring club italiano, f. 437/7 Tassa sui velocipedi, F. Johnson, Per una riduzione della tassa sui velocipedi. Memoriale redatto dal Touring club e indirizzato ai senatori del Regno, 2 dicembre 1905, p. 2.

[30] Cfr. S. Giuntini, Lo sport e la grande guerra. Forze armate e movimento sportivo in Italia di fronte al primo conflitto mondiale, Stato Maggiore dell'Esercito-Ufficio storico Roma, 2000.

[31] I. Bonomi, Lo "sport" e i giovani, in "Avanti!", 29 settembre 1910, citato in S. Pivato, Inno alla gioia. Elogio della fatica, in S. Pivato, L. Veri e N. Cangi (a cura di), In bicicletta: memorie sull'Italia a due ruote, Il Mulino, Bologna 2009, pp. 7-36

[32] Sui ciclisti rossi cfr. P. Colombo e G. Lanotte, La corsa del secolo. Cent'anni di storia italiana attraverso il Giro, Mondadori, Milano 2017, pp. 19-22; A. Lorenzini, I ciclisti rossi. I loro scopi e la loro organizzazione, Ditta Fratelli Cesarani, Bergamo 1913; S. Pivato, La bicicletta e il sol dell'avvenire. Tempo libero e sport nel socialismo della Belle Epoque, Ponte alle Grazie, Firenze 1992, pp. 123-180; Pivato, 2019, pp. 99-115.

[33] Sul Giro d'Italia esiste ormai una letteratura importante. Si possono vedere, tra gli altri: Colombo e Lanotte, 2017; J. Foot, Pedalare! La grande avventura del ciclismo italiano, Rizzoli, Milano 2011; M. Franzinelli, Il Giro d'Italia. Dai pionieri agli anni d'oro, Feltrinelli, Milano 2015; Silei, 2010; D. Marchesini, Coppi e Bartali, Il Mulino, Bologna 1998; D. Marchesini, L'Italia del Giro d'Italia, Il Mulino, Bologna 2009; C. O’Brien, Il Giro d'Italia: una storia di passione, eroismo e fatica, Mondadori, Milano 2017; S. Pivato, Il Giro d'Italia, in M. Isnenghi (a cura di), I luoghi della memoria. Personaggi e date dell'Italia unita, Laterza, Roma-Bari 1997, pp. 333-344.

[34] Cfr. A. Capanni e F. Cervellati, Dal velocipedismo a Gino Bartali. Storia del ciclismo in provincia di Firenze dal XIX secolo al 1931, Provincia di Firenze, Assessorato allo sport, Firenze 2005, pp. 22-25; P. Ciampi, La prima corsa del mondo. Campioni e velocipedi nella Firenze Capitale, Polistampa, Firenze 2012.

[35] Relazione sulla revisione dello Statuto, in "Rivista mensile del Touring Club Ciclistico Italiano", a. VI, n. 3, marzo 1900, pp. 37-38. In realtà già l'anno precedente il sodalizio aveva deliberato «di occuparsi in avvenire di facilitare il turismo anche agli automobilisti»: cfr. L.V. Bertarelli, Il Touring e l'automobilismo, in "Rivista mensile del Touring Club Ciclistico Italiano", n. 6, giugno 1899, p. 12.

[36] Pivato, 2006, pp. 59-67.

[37] L. Pironti, Ordiniamo il nostro caos. Il "codice del pedone”, in "Le vie d'Italia", a. IV, n. 1, gennaio 1920, pp. 32-34.

[38] Per queste statistiche, e per quanto segue, cfr. Belloni, 2019, pp. 133-163.

[39] M. Moraglio, Storia delle prime autostrade italiane (1922-1943). Modernizzazione, affari e propaganda, Trauben, Torino 2007. 
[40] Pivato, 2019, p. 140. 